\title{
Direct Non-adiabatic Simulations of the Photoinduced Charge Transfer Dynamics
}

\author{
Sharma S. R. K. C. Yamijala*,†, ${ }^{*}$ and Pengfei Huo*,† \\ $\dagger$ Department of Chemistry, University of Rochester, 120 Trustee Road, Rochester, New York \\ 14627, United States \\ $\ddagger$ Department of Chemistry, Indian Institute of Technology-Madras, Chennai, India. 600036 \\ E-mail: yamijala@iitm.ac.in; pengfei.huo@rochester.edu
}

\section{Abstract}

We apply direct non-adiabatic dynamics simulations to investigate photoinduced charge transfer reactions. Our approach is based on the mixed quantum-classical fewest switches surface hopping (FSSH) method that treats the transferring electron through time-dependent density functional theory and the nuclei classically. The photoinduced excited state is modeled as a transferring single-electron that initially occupies the LUMO of the donor molecule/moiety. This single-particle electronic wavefunction is then propagated quantum mechanically by solving the time-dependent Schrödinger equation in the basis of the instantaneous molecular orbitals (MOs) of the entire system. The non-adiabatic transitions among electronic states are modeled using the FSSH approach within the classical-path approximation. We apply this approach to simulate the photoinduced charge transfer dynamics in a few well-characterized molecular systems. Our results are in excellent agreement with both the experimental measurements and high-level (yet expensive) theoretical results.

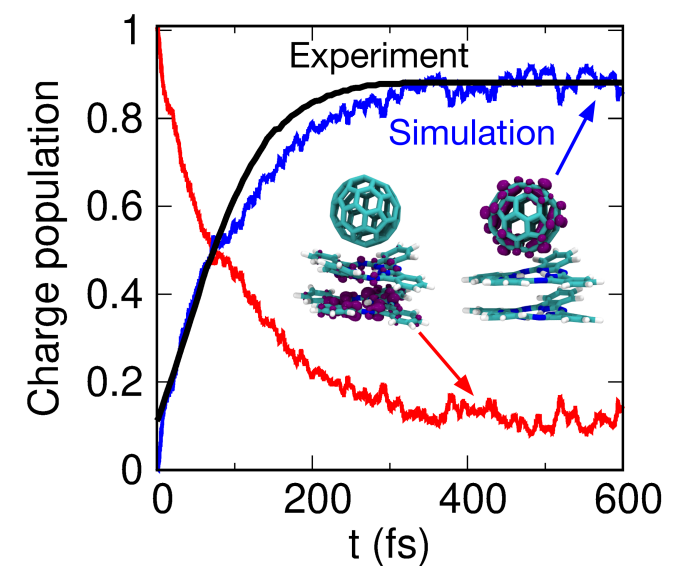

\section{Introduction}

Charge transfer (CT) is a ubiquitous phenomenon that occurs in several chemical reactions, and it plays a fundamental role in many chemical processes. ${ }^{1-4}$ Powering chemical reactions through light-driven processes, rather than through relatively brute-force thermally-activated processes, would be transformative for society. Photoinduced charge transfer (PICT) across the interface between molecules is crucial for determining the reactivity and efficiency of a photochemical system.

$\mathrm{PICT}$ is one of the central reactions that drive solar energy conversion in natural photosynthesis, where the charge-separated state is achieved through multi-photon absorption and involves multiple energy-conversion steps. ${ }^{5-7}$ Like the natural process, there are many artificial systems in which the photoinduced charge transfer plays a crucial role. ${ }^{8-15}$ Examples include PICT in dye-sensitized solar cells, ${ }^{8-11,15-17}$ ultra-fast charge-transfer in organic photovoltaic systems, ${ }^{12-14,18,18-25}$ photocatalytic electron/hole transfer in "colloidal quantum dot-organic molecule complex" interfaces, ${ }^{26-28}$ and photoinduced proton-coupled electron transfer. ${ }^{29-31}$ Understanding the detailed charge transfer dynamics will provide valuable mechanistic insights and design principles for next-generation photocatalytic devices, and profoundly impact energy production and catalysis.

The PICT process is often an intrinsic nonadiabatic process, where the transitions among adiabatic electronic states occur through the coupling between electronic and nuclear degrees of freedom (DOF). Accurately and efficiently simulating the non-adiabatic charge transfer dynamics in largescale systems remains challenging despite encour- 
aging recent progress. ${ }^{32}$ This work presents a simple and accurate approach to investigate the PICT dynamics in model organic molecular systems using on-the-fly simulations.

Our approach uses the fewest switches surface hopping algorithm ${ }^{32,33}$ to capture the influence of nuclear vibrations on the electronic nonadiabatic transitions, and the single-particle timedependent Kohn-Sham (TDKS) approach ${ }^{8,34,35}$ to describe the quantum mechanical state of the transferring electron. With this approach, we investigate the non-adiabatic PICT dynamics in phthalocyanine dimer/fullerene system ${ }^{12,18,36}$ and Carotenoid-Porphyrin- $\mathrm{C}_{60}$ (CPC) triad system, ${ }^{13,19,22,23,37-39}$ which are model systems for understanding the CT dynamics in organic photovoltaics. Our results are in excellent agreement with both experimental measurements and highlevel (yet expensive) theoretical calculations.

\section{Theoretical Approach}

Our approach to obtain the photoinduced chargetransfer dynamics is based on approximately solving the time-dependent Schrödinger equation (TDSE). Specifically, it is based on the mixed quantum-classical fewest switches surface hopping (FSSH) method that treats the transferring electron through the time-dependent density functional theory, and nuclei classically.

The total Hamiltonian of a system can be expressed as

$$
\hat{H}=\hat{T}+\hat{H}_{\mathrm{el}},
$$

where $\hat{T}$ represents the nuclear kinetic energy operator, and $\hat{H}_{\mathrm{el}}$ is the electronic Hamiltonian operator. The adiabatic electronic ground state $\Phi_{0}(\mathbf{R})$, which is the eigenstate of $\hat{H}_{\mathrm{el}}$, as well as the adiabatic energy $E_{0}(\mathbf{R})$, are obtained by solving the following time-independent Schrödinger equation (TISE)

$$
\hat{H}_{\mathrm{el}}\left|\Phi_{0}(\mathbf{R})\right\rangle=E_{0}(\mathbf{R})\left|\Phi_{0}(\mathbf{R})\right\rangle
$$

Among various approaches to solve the above electronic TISE, the Kohn-Sham (KS) Density Functional Theory (DFT) is one of the widely used approaches due to its numerical efficiency. In KS$\mathrm{DFT}$, the single-particle KS equation is given as

$$
\hat{h}_{\mathrm{KS}}\left|\phi_{i}(\mathbf{R})\right\rangle=\epsilon_{i}(\mathbf{R})\left|\phi_{i}(\mathbf{R})\right\rangle
$$

where $\hat{h}_{\mathrm{KS}}$ is the single-particle KS operator, and $\epsilon_{i}(\mathbf{R})$ is the energy of the $i_{\text {th }}$ molecular-orbital $\left|\phi_{i}(\mathbf{R})\right\rangle$. These molecular orbitals are used to construct the ground state (GS) density, ${ }^{40}$ which can be used to compute the ground state energy.

Since solving the TDSE of the total Hamiltonian $\hat{H}$ in Eq. 1 is a challenging task, in this work, we adapt a set of well defined and widely tested approximations. ${ }^{8,10,41}$ A summary of these approximations and their potential limitations are provided in the Supporting Information.

First, we ignore the influence of electronic excitations on the motion of the nuclei, i.e., the nuclear motion is entirely governed by the ground-state potential energy surface, $E_{0}(\mathbf{R}(t))$, through the classical equations of motion, given by

$$
-\nabla_{\mathbf{R}} E_{0}(\mathbf{R})=\mathbf{M} \ddot{\mathbf{R}}
$$

The above approximation, known as the classical path approximation $(\mathrm{CPA})^{41}$ or the neglect of back-reaction (NBR)., ${ }^{42}$ has been extensively tested $^{41-45}$ and is proven to provide reasonable results for photoinduced charge transfer dynamics.

Second, we assumed that the photoinduced electron transfer dynamics can be well-described with a single-particle wavefunction instead of the manyelectron wavefunction. This approximation allows one to use the single-particle version of the time-dependent Kohn-Sham (TDKS) approximation, ${ }^{32,34,35,46}$ where the time-dependent singleparticle excited state $|\Psi(\mathbf{R}(t))\rangle$ can be represented as a linear combination of the ground state KS orbitals $^{10,11}$ as

$$
|\Psi(\mathbf{R}(t))\rangle=\sum_{i} c_{i}(t)\left|\phi_{i}(\mathbf{R}(t))\right\rangle
$$

where $\left\{c_{i}(t)\right\}$ are the time-dependent expansion coefficients. The single-particle approximation of the TDKS equation has shown to accurately describe the charge-transfer dynamics $8,10,37,47,48$ and excitation energy transfer process. ${ }^{49}$ It has been extensively tested against more accurate linear-response (LR)-TDDFT calculations. ${ }^{35}$ Note that our eventual goal for this implementation is to investigate mesoscopic systems such as Bulk Heterojunctions that contain $10^{3}-10^{4}$ atoms. For these systems calculating the excited states through LR-TDDFT are extremely expensive and beyond the practically available computational resources, even with the DFTB approximation. ${ }^{50}$ TDKS, on the other hand, has proven to be as accurate as (LR)-TDDFT cal- 
culations $^{35}$ for a variety of systems, with the numerical cost that is comparable to a ground state KS calculations. Hence, we choose TDKS in this study.

Within the single-particle TDKS approximation, $|\Psi(\mathbf{R}(t))\rangle$ satisfies the following equation ${ }^{46}$

$$
i \hbar \frac{\partial}{\partial t}|\Psi(\mathbf{R}(t))\rangle=\hat{h}_{\mathrm{KS}}(\mathbf{R}(t))|\Psi(\mathbf{R}(t))\rangle .
$$

Plugging Eq. 5 into Eq. 6, and multiplying the resulting expression with $\left\langle\phi_{j}(t)\right|$ gives us the following differential equation for the time-dependent expansion coefficients

$$
\dot{c}_{j}(t)=-\frac{i}{\hbar} c_{j}(t) \epsilon_{j}(\mathbf{R}(t))-\sum_{i} c_{i}(t)\left\langle\phi_{j}(t) \mid \frac{\partial}{\partial t} \phi_{i}(t)\right\rangle,
$$

where we used shorthand notation $\left|\phi_{i}(t)\right\rangle \equiv$ $\left|\phi_{i}(\mathbf{R}(t))\right\rangle$. The non-adiabatic couplings $\left\langle\phi_{j} \mid \frac{\partial}{\partial t} \phi_{i}\right\rangle$ are responsible for electronic transitions among the single-particle adiabatic states. They are related to the derivative coupling vectors through $\left\langle\phi_{j}(\mathbf{R}(t)) \mid \frac{\partial}{\partial t} \phi_{i}(\mathbf{R}(t))\right\rangle=\left\langle\phi_{j}(\mathbf{R}(t))|\nabla| \phi_{i}(\mathbf{R}(t))\right\rangle \dot{\mathbf{R}}$.

Third, we we adapt the fewest-switches surface hopping (FSSH) algorithm within the CPA approximation $^{41,45}$ to describe the electronic nonadiabatic transitions induced by the motion of the nuclei. Solving Eq. 7 provides $\left\{c_{j}(t)\right\}$, and substituting them in Eq. 5 gives the time-dependent single-particle electronic wavefunction, from which one can construct the time-dependent charge transfer density. ${ }^{10,11}$ However, due to the lack of microscopic feedback from the electronic subsystem to the nuclear subsystem, directly using the above $c_{j}(t)$ leads to an incorrect population dynamics ${ }^{51}$ that corresponds to an infinite temperature of the electronic subsystem, leading to the artificial equal population among all single-particle electronic states, ${ }^{51}$ which is demonstrated in the Supporting Information. To alleviate this problem, and to accurately describe the non-adiabatic transitions among single-particle electronic states, we adapt the fewest-switches surface hopping (FSSH) algorithm within the CPA approximation. ${ }^{41,45}$

During CPA-FSSH dynamics, ${ }^{41,45}$ an instantaneous active state is assigned to the system, and the probability of switching from the current state $\left|\phi_{i}(\mathbf{R}(t))\right\rangle$ to any other state $\left|\phi_{j}(\mathbf{R}(t))\right\rangle$ during the time-step $t \in[t, t+\delta]$ is given by ${ }^{41,45}$

$$
\tilde{g}_{i j}(t)=-\frac{2 \operatorname{Re}\left[c_{i}^{*}(t) c_{j}(t)\left\langle\phi_{j}(t) \mid \frac{\partial}{\partial t} \phi_{i}(t)\right\rangle\right]}{c_{i}^{*}(t) c_{i}(t)} \delta t,
$$

where $\rho_{i j}(t)=c_{i}^{*}(t) c_{j}(t)$ are the adiabatic electronic density matrix elements. In the original FSSH formalism, ${ }^{33}$ nuclear velocities are rescaled to conserve the total energy after switching the active states. Within the $\mathrm{CPA}^{9,41}$ where the backreaction on the nuclei is neglected, the transition probabilities are rescaled as follows

$$
g_{i j}(t)=\max \left[\tilde{g}_{i j}(t) b_{i j}(t), 0\right]
$$

where $b_{i j}(t)=e^{-\left(\epsilon_{j}-\epsilon_{i}\right) / \mathrm{k}_{\mathrm{B}} T}$ for $\epsilon_{j}>\epsilon_{i}$, and $b_{i j}(t)=$ 1 for $\epsilon_{j} \leq \epsilon_{i}, \epsilon_{i}(\mathbf{R}(t))$ and $\epsilon_{j}(\mathbf{R}(t))$ are the orbital energies obtained by solving Eq. 3 with the nuclear configuration $\mathbf{R}(t), \mathrm{k}_{\mathrm{B}}$ is the Boltzmann constant, and $T$ is the temperature of the system, which is assumed to be constant during the non-adiabatic dynamics.

A switch from state $\left|\phi_{i}(\mathbf{R})\right\rangle$ to state $\left|\phi_{j}(\mathbf{R})\right\rangle$ takes place when

$$
\sum_{k=1}^{k=j-1} g_{i k}<\xi \leq \sum_{k=1}^{k=j} g_{i k},
$$

where $\xi$ is a uniform random number between 0 and 1. An ensemble of nuclear trajectories are generated by propagating the nuclei with Eq. 4, whereas one or more CPA-FSSH trajectories are generated on top of $R(t)$ by solving Eq. 7 to obtain the time-dependent electronic expansion coefficients, and then active state is determined based on Eq. 10. The above procedure is essentially the same as those described in Ref. 34, apart from a few technical differences. First, we use an accurate charge population estimators (Eq. 14) as well as the reduced density matrix population estimator (Eq. 11). To the best of our knowledge, this is the first time that both are used in direct charge transfer CPA-FSSH simulations. Second, we implement the CPA-FSSH approach directly within the DFTB+ software package. For this reason, we do not have to store any elements such as eigenvalues, eigenvectors, derivative couplings, etc, which will significantly speed up the simulations for the system with a large size (eg, thousands of atoms).

We note that FSSH could potentially create an artificial electronic coherence, and a decoherence correction is employed in such circumstances. ${ }^{33,52}$ Also, many decoherence approaches are already available for CPA-FSSH. ${ }^{32,41,43,53}$ In this work, we choose not to use any decoherence correction because CPA-FSSH already provided an accu- 
rate charge transfer dynamics compared to experiments. We compute the adiabatic reduced density matrix as

$$
\bar{\rho}_{i j}(t)=\left\langle\rho_{i j}(t)\right\rangle
$$

where $\langle\ldots\rangle$ represents the ensemble average over the CPA-FSSH trajectories. The estimator $\rho_{i j}(t)$ is expressed as follows ${ }^{54}$

$$
\begin{aligned}
& \rho_{i i}(t)=\left\langle\phi_{i}(R(t)) \mid \phi_{\alpha}(R(t))\right\rangle=\delta_{i \alpha} \\
& \rho_{i j}(t)=c_{i}^{*}(t) c_{j}(t) \quad(\text { for } i \neq j) .
\end{aligned}
$$

where, $\left|\phi_{\alpha}(\mathbf{R}(t))\right\rangle$ is the active state at time $t$ along a nuclear trajectory $\mathbf{R}(t)$, and $c_{j}(t)$ are the corresponding expansion coefficients. The diagonal matrix elements $\rho_{i i}(t)$ are chosen based on the active state $\left|\phi_{\alpha}(R(t))\right\rangle$. Thus, along a specific nuclear trajectory $R(t)$, the instantaneous population at time $t$ is considered to be 1 for the active state $\left|\phi_{\alpha}(R(t))\right\rangle$, and 0 for all the other states.

To characterize the photoinduced charge transfer dynamics in a given system, we compute the time-dependent charge populations on each moiety (such as a donor or acceptor) of the system. The charge population on a specific fragment is obtained by projecting the adiabatic reduced density matrix onto the Atomic Orbital (AO) basis associated with that molecular moiety $\mathcal{N}$ as follows ${ }^{10,11}$

$P_{\mathcal{N}}(t)=\operatorname{Re}\left[\sum_{\mu \in \mathcal{N}}^{\nu} \sum_{i j} \rho_{i j}(t) C_{\mu i}(\mathbf{R}(t)) S_{\mu \nu} C_{\nu j}(\mathbf{R}(t))\right]$,

where $S_{\mu \nu}=\left\langle\varphi_{\mu}(\mathbf{r}, \mathbf{R}(t)) \mid \varphi_{\nu}(\mathbf{r}, \mathbf{R}(t))\right\rangle$ is the AO overlap matrix, and $\left\{C_{\mu i}(\mathbf{R}(t))\right\}$ are the AO expansion coefficients obtained by solving Eq. 3 in the AO basis (through the self-consistent procedure ${ }^{55}$ ) as follows

$$
\left|\phi_{i}(\mathbf{R}(t))\right\rangle=\sum_{\mu} C_{\mu i}(\mathbf{R}(t))\left|\varphi_{\mu}(\mathbf{r}, \mathbf{R})\right\rangle .
$$

Note that in Eq. 14, we choose to use the active state estimator (Eq. 12) for the adiabatic electronic population and the electronic estimator (Eq. 13) for the adiabatic electronic coherence. This choice has shown to provide the most accurate diabatic population. ${ }^{54}$ The expectation value of the charge population is obtained as

$$
\bar{P}_{\mathcal{N}}(t)=\left\langle P_{\mathcal{N}}(t)\right\rangle
$$

where $\langle\ldots\rangle$ represents the ensemble average over the CPA-FSSH trajectories, and $P_{N}(t)$ is the charge population estimator expressed in Eq. 14.

\section{Computational Details}

All electronic structure calculations are performed using the semi-empirical Density Functional TightBinding (DFTB) method, ${ }^{55-57}$ which is an approximation to the KS-DFT. Earlier works have demonstrated that DFTB provides accurate electronic structure for studying charge transfer dynamics in various organophotovoltaic complexes. ${ }^{38,58,59}$ More specifically, DFTB3 method, ${ }^{57}$ which is a thirdorder Taylor expansion of the DFT total energy around a reference density, is used in this work. DFTB3 is proven to be a well-suited method ${ }^{57}$ to describe the charges in systems with $\mathrm{C}, \mathrm{H}, \mathrm{N}, \mathrm{O}$, and $\mathrm{P}$ elements, with improved Coulomb interaction between atomic partial charges. ${ }^{57}$ Electronic structure calculations in this work are performed with the DFTB3 method and 3ob-3-1 Slater-Koster parameter set ${ }^{60}$ as implemented in the 17.1 version of the DFTB + package. ${ }^{61}$ We also included the dispersion interactions using the Lennard-Jones dispersion model with the UFF parameters ${ }^{62}$ as implemented in the DFTB+ code.

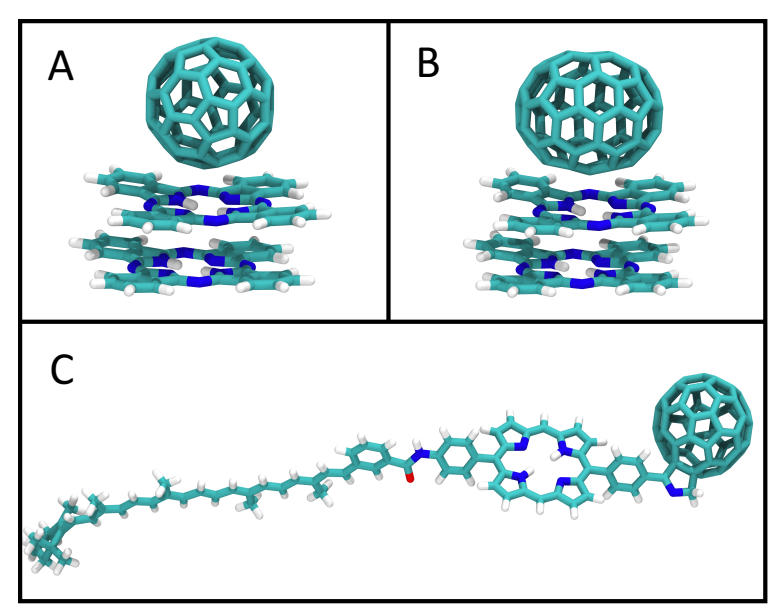

Figure 1: Structures of the model systems investigated in this paper: (A) $2 \mathrm{H}_{2} \mathrm{Pc} / \mathrm{C}_{60}$ (B) $2 \mathrm{H}_{2} \mathrm{Pc} / \mathrm{C}_{70}$ and $(\mathrm{C})$ carotenoid-porphyrin- $\mathrm{C}_{60}(\mathrm{CPC})$ triad. All geometries presented in this figure are optimized at the DFTB3 level of theory.

The initial nuclear geometries are obtained according to the ground state canonical ensemble, through the following procedure. First, the system is equilibrated in the NVT ensemble for 100 ps with a nuclear time-step $d t=0.5 \mathrm{fs}$, using the Nosé-Hoover chain thermostat as implemented in the DFTB+ package. From this NVT trajectory, 
we collected 50 different initial conditions (coordinates and velocities at every $2 \mathrm{ps}$ interval) for the subsequent non-adiabatic dynamics propagation.

The initial electronic excitation $|\Psi(\mathbf{r}, 0)\rangle$ is modeled as the LUMO of the donor moiety $\left|\phi_{\mathrm{LUMO}}^{\mathrm{D}}\right\rangle$, which is a widely used approximation for simulating photoinduced charge transfer dynamics. ${ }^{10,11}$ This choice provides a reasonable single-electron picture of the localized photo-excitation in the system. Here, the LUMO of the donor moiety is obtained from a separate DFTB3 calculation performed for the isolated donor moiety. ${ }^{10,11,37,63}$ The initial coefficients for the electronic states are computed as $c_{i}(0)=\left\langle\phi_{i}\left(\mathbf{R}(0)\left|\phi_{\mathrm{LUMO}}^{\mathrm{D}}\right\rangle=\right.\right.$ $\sum_{\mu} C_{\mu i}(\mathbf{R}(0))\left\langle\varphi_{\mu}(\mathbf{R}(0))\left|\sum_{\nu} C_{\nu}^{\mathrm{D}}(\mathbf{R}(0))\right| \varphi_{\nu}(\mathbf{R}(0))\right\rangle=$ $\sum_{\mu \nu} C_{\mu i}(\mathbf{R}(0)) S_{\mu \nu}(\mathbf{R}(0)) C_{\nu}^{\mathrm{D}}(\mathbf{R}(0))$, which is evaluated with zero expansion coefficients over the AOs on the acceptor moiety. The active state at $t=0$ in the CPA-FSSH calculation is chosen stochastically among $\mid \phi_{i}(\mathbf{R}(0)\rangle$ based upon the probability of $\left|c_{i}(0)\right|^{2}$.

For the CPA-FSSH non-adiabatic dynamics simulations, the nuclear propagation is performed using the velocity-Verlet integrator with a time-step of $d t=0.1$ fs under the NVE ensemble. Adapting the classical path approximation (CPA), ${ }^{9-11,41}$ we used the ground state DFTB3 gradients (cf. Eq. 4) during the nuclear propagation. To obtain the time-dependent electronic expansion coefficients, we numerically integrated Eq. 7 using the fourthorder Runge-Kutta algorithm with a time-step of $d t=10^{-3}$ fs. To compute the time-dependent nonadiabatic couplings, we adapted a well-established finite difference approximation ${ }^{64}$ as follows

$$
\left\langle\phi_{j} \mid \frac{\partial}{\partial t} \phi_{i}\right\rangle=\frac{1}{2 \tau}\left[\left\langle\phi_{j}(t) \mid \phi_{i}(t+\tau)\right\rangle-\left\langle\phi_{j}(t+\tau) \mid \phi_{i}(t)\right\rangle\right],
$$

where the MO overlaps $\left\langle\phi_{j}(t+\tau) \mid \phi_{i}(t)\right\rangle$ at different time-steps needs to be carefully calculated by following the random phases of the MOs generated from the electronic structure calculations. ${ }^{65-67} \mathrm{Ex}$ pressing the MOs expanded as linear combinations of the atomic orbitals (AOs), i.e., $\left|\phi_{i}(\mathbf{R}(t))\right\rangle=$ $\left.\sum_{\mu} C_{\mu i}(\mathbf{R}(t)) \mid \varphi_{\mu}(\mathbf{R})\right)$, the overlap between two MOs in Eq. 17 can be computed as

$$
\begin{aligned}
& \left\langle\phi_{j}(\mathbf{R}(t+\tau)) \mid \phi_{i}(\mathbf{R}(t))\right\rangle \\
& =\sum_{\mu \nu} C_{\mu j}(\mathbf{R}(t+\tau)) C_{\nu i}(\mathbf{R}(t)) S_{\mu \nu}(t+\tau, t)
\end{aligned}
$$

where, $S_{\mu \nu}(t+\tau, t)$ is the overlap between two atomic orbitals (AOs) at two different time-steps

$$
S_{\mu \nu}(t+\tau, t)=\left\langle\varphi_{\mu}(\mathbf{r}, \mathbf{R}(t+\tau)) \mid \varphi_{\nu}(\mathbf{r}, \mathbf{R}(t))\right\rangle .
$$

These AO overlap integrals are explicitly evaluated with our in-house version of DFTB + code.

A total of 50 initial nuclear configuration is used for the ensemble average, and for each nuclear configuration, a total of $10^{4} \mathrm{CPA}-\mathrm{FSSH}$ trajectories are used. Expectation values are calculated through the ensemble average over specific CPAFSSH trajectories on top of each nuclear trajectory.

Fig. 1 depicts the optimized geometries of the model system considered in this work: (A) $2 \mathrm{H}_{2} \mathrm{Pc}_{6} / \mathrm{C}_{60}$ (B) $2 \mathrm{H}_{2} \mathrm{Pc} / \mathrm{C}_{70}$ and $(\mathrm{C})$ carotenoidporphyrin-fullerene $\left(\mathrm{CPC}_{60}\right)$ triad. The Cartesian coordinates of the optimized nuclear geometries (at the level of DFTB3) are provided in the supporting information. For the $2 \mathrm{H}_{2} \mathrm{Pc} / \mathrm{C}_{60}$ and $2 \mathrm{H}_{2} \mathrm{Pc} / \mathrm{C}_{70}$ model systems, we considered $\mathrm{H}_{2} \mathrm{Pc}$ dimer as the donor, ${ }^{12}$ and for the CPC triad, porphyrin is considered as the donor moiety. ${ }^{37}$ To reduce the computational cost, we only consider a finite size of CPA-FSSH active space that includes the LUMO to $\mathrm{LUMO}+9$ orbitals of the entire system, which are the low-lying orbitals that participate directly in the photoinduced charge transfer process. We carefully checked that the non-adiabatic dynamics is confined within this subspace, i.e., further increasing the active space does not influence the dynamics. 68

\section{Results ans Discussions}

Fig. 2 presents the time dependent non-adiabatic dynamics of the $2 \mathrm{H}_{2} \mathrm{Pc} / \mathrm{C}_{60}$ system with a specific nuclear trajectory through the CPA-FSSH simulation. Fig. 2A depicts the transferring charge density of the active state along a given nuclear trajectory at $t=32.2 \mathrm{fs}, t=119.6 \mathrm{fs}$, and $t=150$ $\mathrm{fs}$, respectively. The initial charge density is sensitive to the nuclear geometries, which can either localize on one of the $\mathrm{H}_{2} \mathrm{Pc}$ molecules, or delocalize over the $\mathrm{H}_{2} \mathrm{Pc}$ dimer. An additional analysis is provided in the supporting information. Fig. 2B presents the time-dependent orbital energies of $\left|\phi_{i}(\mathbf{R}(t))\right\rangle(\mathrm{LUMO}$ to LUMO+6) of this system. As shown in the figure, the MO orbital energies come close to each other at various instances of time, forming avoided crossings where the nonadiabatic coupling element between the surfaces would be large and resulting in a higher proba- 


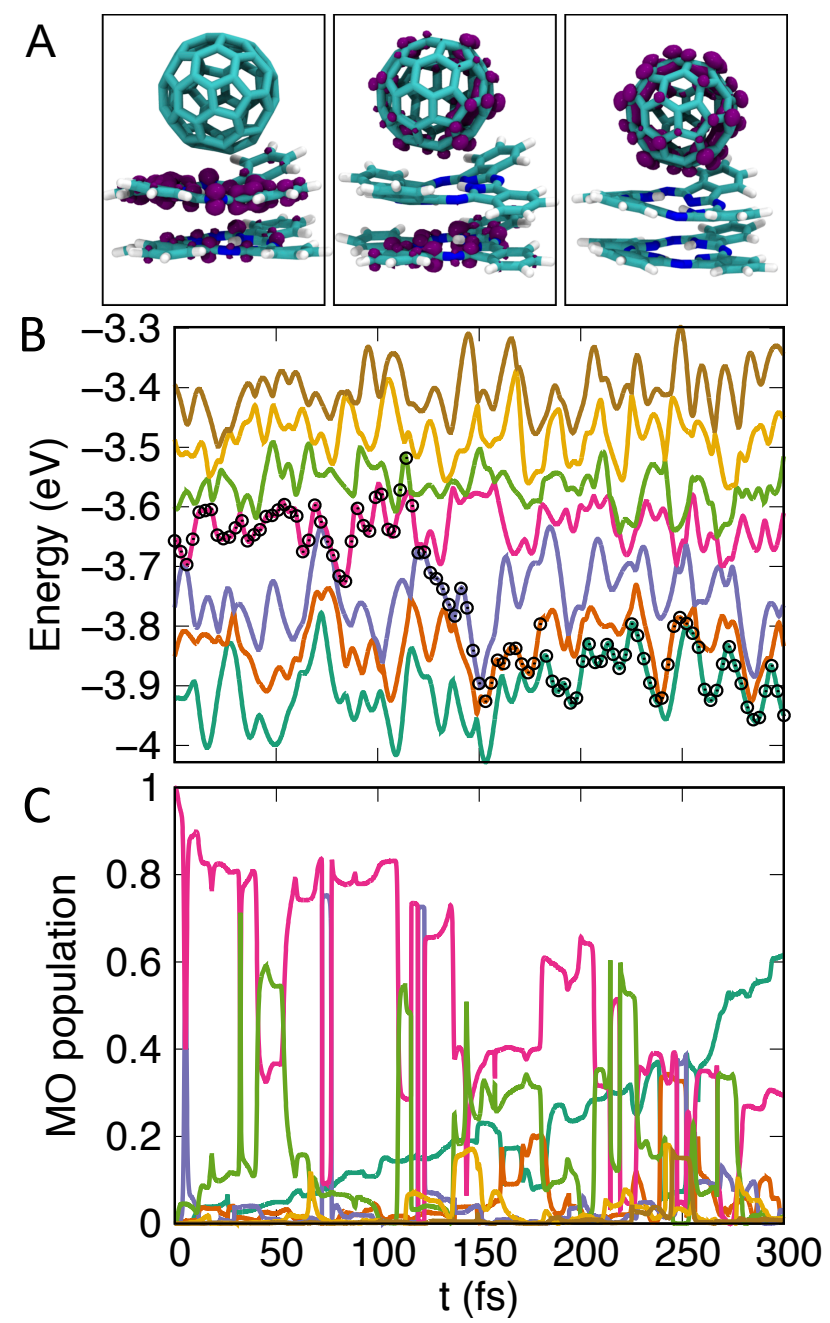

Figure 2: A. Charge density of the active state along a given nuclear trajectory of the phthalocyanine dimer/fullerene $\left(2 \mathrm{H}_{2} \mathrm{Pc} / \mathrm{C}_{60}\right)$ system, at $t=32.2 \mathrm{fs}$, $t=119.6 \mathrm{fs}$, and $t=150 \mathrm{fs}$, respectively. B. The timedependent energies of $\left|\phi_{i}(\mathbf{R}(t))\right\rangle$ (LUMO to LUMO+6) along a give nuclear trajectory. The time-dependent active states for a given CPA-FSSH trajectory are indicated with black open circles. C. The adiabatic (MO) populations (Eq. 11) for the same nuclear trajectory, averaged over 10,000 CAP-FSSH trajectories (with the corresponding color coding of the MOs in panel $\mathbf{B}$ for $\mathrm{LUMO}$ to $\mathrm{LUMO}+6$.)

bility for the active state to hop from one to the other (see Eq. 8). The character of the activate state changes due to both the motion of the nuclei (from the first to the second panel in Fig. 2A) as well as non-adiabatic transitions among states (from the second to the third panel in Fig. 2A). The former and the latter ways are classified as the adiabatic vs. non-adiabatic contribution of the charge transfer. ${ }^{69}$ Fig. $2 \mathrm{C}$ presents the time-dependent $\mathrm{MO}$ electronic population of the $2 \mathrm{H}_{2} \mathrm{Pc} / \mathrm{C}_{60}$ system along one specific nuclear trajectory, computed through the ensemble average of $10^{4}$ CPA-FSSH trajectories through Eq. 11, color coded according to the MOs presented in Fig. 2B. The nonadiabatic transitions occur at the avoid crossings in Fig. 2B.
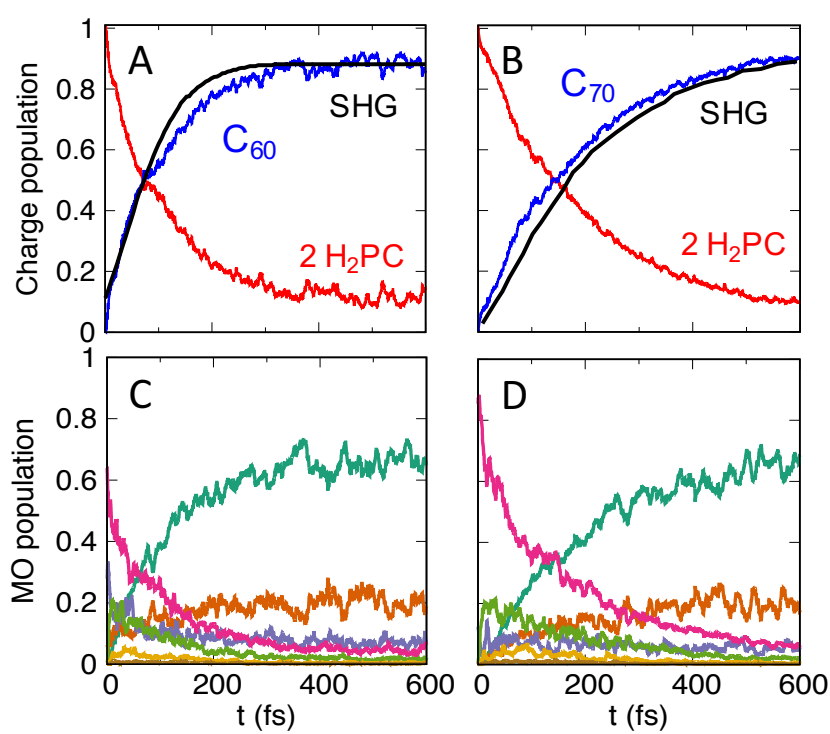

Figure 3: (A) Charge population on $\mathrm{C}_{60}$ (blue), on $2 \mathrm{H}_{2} \mathrm{Pc}$ (red), as well as the SHG experimental signal (black) adapted from Ref. 12 that indicate the timedependent charge density on $\mathrm{C}_{60}$. (B) Time-dependent charge population of the $2 \mathrm{H}_{2} \mathrm{Pc} / \mathrm{C}_{70}$ system. (C) and (D) presents the $\mathrm{MO}$ populations of $2 \mathrm{H}_{2} \mathrm{Pc} / \mathrm{C}_{60}$ and $2 \mathrm{H}_{2} \mathrm{Pc} / \mathrm{C}_{70}$, respectively, with the color coding corresponds to the energy levels in Fig. $2 \mathrm{~b}$.

Fig. 3 presents the population dynamics obtained over an ensemble average of 50 nuclear trajectories with $10^{4} \mathrm{CPA}-\mathrm{FSSH}$ trajectories on top of each nuclear trajectory. Fig. 3A presents the charge population $P_{\mathcal{N}}(t)$ (Eq. 14) of the donor molecules $2 \mathrm{H}_{2} \mathrm{Pc}$ (red) and the acceptor molecule $\mathrm{C}_{60}$ (blue) in the $2 \mathrm{H}_{2} \mathrm{Pc} / \mathrm{C}_{60}$ system. To assess the accuracy of our direct simulations, the experimentally measured time-dependent charge population on fullerene, which is based on transient second harmonic generation ( $\mathrm{SHG}$ ) spectroscopy, ${ }^{12}$ is presented (black curve). Our theoretical results almost quantitatively reproduce the experimental measurements, as well as FSSH simulation using configuration-interaction Singles-Doubles (CISD) with Pariser-Parr-Pople (PPP) level of theory, ${ }^{12}$ suggesting a reasonable accuracy and reliability of the current simulation approach. On the other hand, due to the large number of approximations made in our current theoretical approach (including the propagation of nuclei on the ground electronic state, the single-particle treatment, and the 


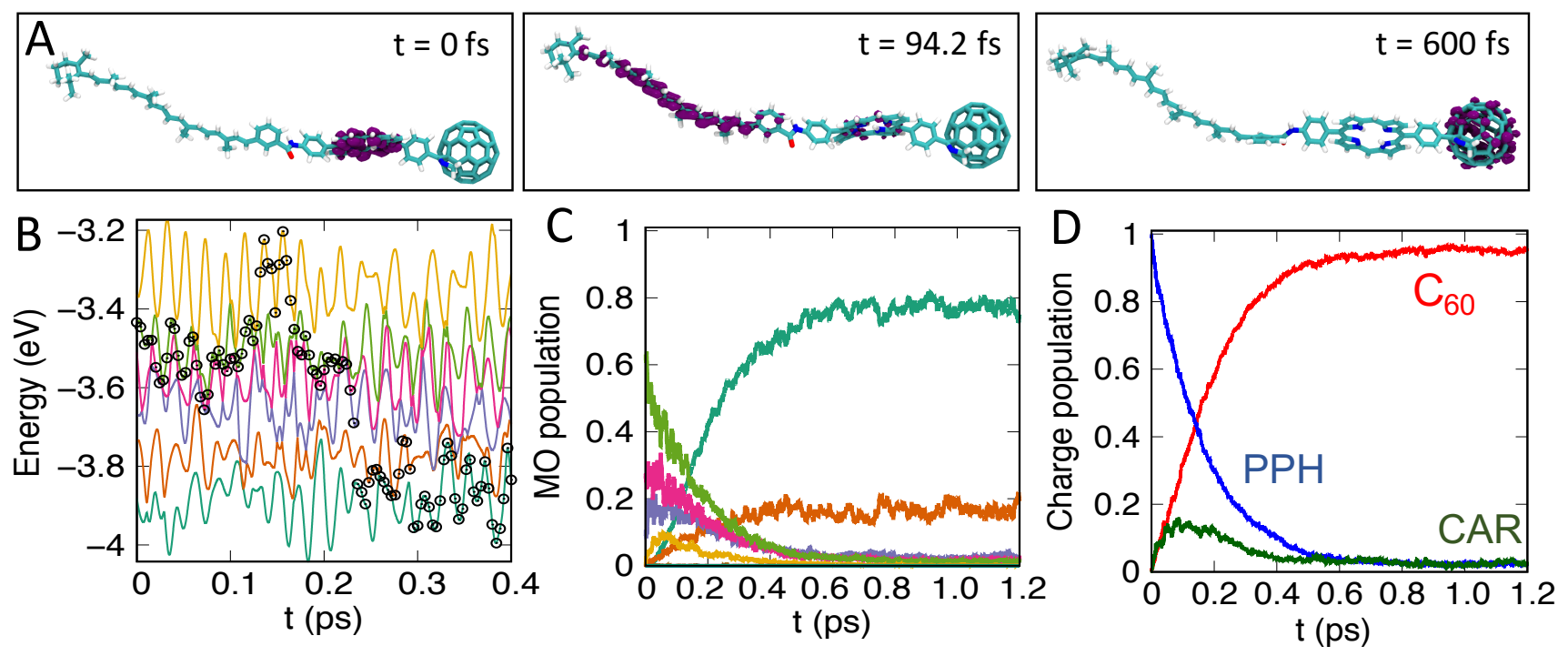

Figure 4: Photoinduced charge transfer dynamics of the CPC triad. (A) Charge density of the active state MO at time t. (B) Time-dependent MO energies and the active states (black open circles), (C) MO populations, (D) time-dependent charge population on each moiety.

ignorance of decoherence corrections in the FSSH approach), it is also possible that a large amount of error cancellations that leads to accurate results in the end.

Fig. 3B presents the charge population of the donor molecules $2 \mathrm{H}_{2} \mathrm{Pc}$ (red) and the acceptor molecule $\mathrm{C}_{70}$ (blue) in the $2 \mathrm{H}_{2} \mathrm{Pc} / \mathrm{C}_{70}$ system. This simulation successfully predicts a slower charge transfer dynamics in $2 \mathrm{H}_{2} \mathrm{Pc} / \mathrm{C}_{70}$ system compared to $2 \mathrm{H}_{2} \mathrm{Pc} / \mathrm{C}_{60}$, as observed in the $\mathrm{SHG}$ experiments. Fig. 3C-D presents the corresponding MO population (computed from Eq. 11) associated with $2 \mathrm{H}_{2} \mathrm{Pc} / \mathrm{C}_{60}$ and $2 \mathrm{H}_{2} \mathrm{Pc} / \mathrm{C}_{70}$, respectively, with the same color coding for the MO orbitals used for LUMO to LUMO+6 in Fig. $2 \mathrm{~B}$.

Fig. 4 presents the CT dynamics in the $\mathrm{CPC}$ triad model system, which is another wellstudied prototypical artificial light-harvesting system. ${ }^{13,19,37,39}$ Earlier experimental investigations ${ }^{70,71}$ have shown photoinduced charge separation in this system. Recent theoretical work suggests that the simple Marcus theory is unable to properly predict the photoinduced electrontransfer time-scales in this system. ${ }^{13,39}$ Here, we use direct non-adiabatic simulations to investigate the charge transfer dynamics. Fig. 4A, presents the time-dependent transferring charge density of the corresponding active state along a given nuclear trajectory at $t=0 \mathrm{fs}, t=94.2 \mathrm{fs}$, and $t=600 \mathrm{fs}$, respectively. Fig. $4 \mathrm{~B}$ presents the time-dependent orbital energies of $\left|\phi_{i}(\mathbf{R}(t))\right\rangle$ (LUMO to LUMO+6) of this system. Fig. 4C presents the corresponding electronic populations of each MO (based on Eq. 11), and Fig. 4D presents the charge population (based on Eq. 14) for the PPH (blue), CAR (green), and the $\mathrm{C}_{60}$ (red) moiety. The population dynamics is obtained from the ensemble average of $10^{4}$ CPA-FSSH trajectories per nuclear trajectory, and 50 nuclear trajectories in total.

Our simulations suggest a significant amount $(90 \%)$ of charge transfer from the porphyrin moiety to the $\mathrm{C}_{60}$ occurring within $\sim 0.4$ ps time-scale, and a complete charge transfer to the $\mathrm{C}_{60}$ moiety within $\sim 0.6$ ps time-scale. Earlier theoretical works in related systems (with a few functional groups on the porphyrin and the Fullerene moieties) where the predicted $\mathrm{CT}$ timescales are spanned over a range of $70 \mathrm{fs}^{19}$ (when completely ignore the motion of nuclei) to $3-5$ ps. ${ }^{13,37,38}$ In a combined experimental and computational study, it has shown that the formation of $[\mathrm{C}-\mathrm{P}]^{+}-\mathrm{C}_{60}^{-}$within $70 \mathrm{fs}^{19}$ On the other hand, for a synthesized the CPC triad, the reported the formation of the $\mathrm{CT}$ state is within in $10 \mathrm{ps}$ in 2-Methyltetrahydrofuran solvent. ${ }^{70}$ Recent work based on the Linearized Semiclassical Nonequilibrium Fermi's Golden Rule (as well as the instantaneous Marcus theory) ${ }^{39}$ suggest that $90 \%$ of the CT occurs during the first 0.6 ps of the simulation for the bent configuration of CTC, agreeing with what we find from our direct simulation. Together, all the above results clearly demonstrate that the current direct simulation approach is both 
efficient and accurate to investigate the photoinduced charge transfer dynamics.

\section{Conclusions}

This work presents the direct non-adiabatic simulation of photoinduced charge transfer reaction in well-studied phthalocyanine/fullerene and carotenoid-porphyrin-fullerene systems. We demonstrate that our approach can predict the timescales of CT dynamics that are in wellagreement with both experimental results ${ }^{12}$ and theoretical studies. ${ }^{12,39}$ Due to the computationally inexpensive nature of the time-dependent DFT with the semi-empirical DFTB calculations, the DFTB-CPA-FSSH approach shows promise for investigating the CT dynamics in large systems.

Despite the success of the current approach in simulating the model systems presented in this work, we acknowledge its potential limitations, including (i) the single-particle picture that could fail for the strongly coupled electron and hole dynamics, ${ }^{22,36,37,41,72}$ (ii) the validity of the classical path approximation, ${ }^{47,53,63,73}$ and (iii) the accuracy of the FSSH algorithms. ${ }^{43,52}$ Encouraging progress is being made to address each of the above three challenges, enabling the possibility to obtain a more accurate description of the charge transfer dynamics in large complex systems.

\section{Acknowledgement}

This work was supported by the National Science Foundation "Collaborative Research: Investigating Photoinduced Charge Transfer Dynamics Across MoleculeNanocrystal Interfaces" under the Grant number CHE1900125, as well as by the University of Rochester startup funds. Computing resources were provided by the Center for Integrated Research Computing (CIRC) at the University of Rochester. We would also like to thank Prof. Josef Michl for inspiring our work in the field of singlet fission, charge transfer in organic molecules, and beyond.

\section{Supporting Information Available}

The Supporting Information is available free of charge on the ACS Publications website. Cartesian coordinates of the optimized nuclear geometries for systems considered in this work; a pseudo-code of our DFTBCPA-FSSH approach; and a table listing all the major approximations that we considered in this work.

\section{References}

(1) Marcus, R. A. Electrostatic free energy and other properties of states having nonequilibrium polarization. I. J. Chem. Phys. 1956, 24, 979-989.

(2) Marcus, R. A. On the theory of oxidationreduction reactions involving electron transfer. I. J. Chem. Phys. 1956, 24, 966-978.

(3) others,, et al. Charge migration and charge transfer in molecular systems. Struct. Dyn. 2017, 4, 061508 .

(4) May, V.; Kühn, O. Charge and energy transfer $d y$ namics in molecular systems; John Wiley \& Sons, 2008.

(5) Blankenship, R. E. Molecular mechanisms of photosynthesis; John Wiley \& Sons, 2014.

(6) Barber, J.; Tran, P. D. From natural to artificial photosynthesis. Journal of The Royal Society Interface 2013, 10, 20120984.

(7) Rivalta, I.; Brudvig, G. W.; Batista, V. S. Oxomanganese complexes for natural and artificial photosynthesis. Current opinion in chemical biology 2012, 16, 11-18.

(8) Duncan, W. R.; Prezhdo, O. V. Theoretical studies of photoinduced electron transfer in dyesensitized TiO2. Annu. Rev. Phys. Chem. 2007, $58,143-184$.

(9) Duncan, W. R.; Craig, C. F.; Prezhdo, O. V. Time-Domain ab Initio Study of Charge Relaxation and Recombination in Dye-Sensitized TiO2. J. Am. Chem. Soc. 2007, 129, 8528-8543.

(10) Rego, L. G.; Batista, V. S. Quantum Dynamics Simulations of Interfacial Electron Transfer in Sensitized TiO2 Semiconductors. J. Am. Chem. Soc. 2003, 125, 7989-7997.

(11) Abuabara, S. G.; Rego, L. G. C.; Batista, V. S. Influence of Thermal Fluctuations on Interfacial Electron Transfer in Functionalized TiO2 Semiconductors. J. Am. Chem. Soc. 2005, 127, 1823418242 .

(12) Jailaubekov, A. E.; Willard, A. P.; Tritsch, J. R.; Chan, W.-L.; Sai, N.; Gearba, R.; Kaake, L. G.; Williams, K. J.; Leung, K.; Rossky, P. J.; Zhu, X.Y. Hot Charge-Transfer Excitons Set the Time Limit for Charge Separation at Donor/Acceptor 
Interfaces in Organic Photovoltaics. Nat. Mater. 2013, 12, 66-73.

(13) Sun, X.; Zhang, P.; Lai, Y.; Williams, K. L.; Cheung, M. S.; Dunietz, B. D.; Geva, E. Computational Study of Charge-Transfer Dynamics in the Carotenoid-Porphyrin-C60 Molecular Triad Solvated in Explicit Tetrahydrofuran and Its Spectroscopic Signature. J. Phys. Chem 2018, 122, 1128811299 .

(14) Coropceanu, V.; Chen, X.-K.; Wang, T.; Zheng, Z.; Brédas, J.-L. Charge-transfer electronic states in organic solar cells. Nat. Rev. Mater. 2019, 1-19.

(15) Akimov, A. V.; Neukirch, A. J.; Prezhdo, O. V. Theoretical insights into photoinduced charge transfer and catalysis at oxide interfaces. Chem. Rev. 2013, 113, 4496-4565.

(16) Torres, A.; Oliboni, R. S.; Rego, L. G. Vibronic and Coherent Effects on Interfacial Electron Transfer Dynamics. J. Phys. Chem. Lett. 2015, 6, 4927-4935.

(17) Sriparna Mukherjee, D. N. B.; Jakubikova, E. Cyclometalated Fe(II) Complexes as Sensitizers in Dye-Sensitized Solar Cells. Inorg. Chem. 2015, 54, 560569 .

(18) Lee, M.; Geva, E.; Dunietz, B. Donor-to-Donor vs. Donor-to-Acceptor Interfacial Charge Transfer States in the Phthalocyanine-Fullerene Organic Photovoltaic System. J. Phys. Chem. Lett. 2014, 5, 3810-3816.

(19) Rozzi, C. A.; Falke, S. M.; Spallanzani, N.; Rubio, A.; Molinari, E.; Brida, D.; Maiuri, M.; Cerullo, G.; Schramm, H.; Christoffers, J.; Lienau, C. Quantum coherence controls the charge separation in a prototypical artificial lightharvesting system. Nat. Comm 2013, 4, 1602.

(20) Tamura, H.; Burghardt, I. Ultrafast Charge Separation in Organic Photovoltaics Enhanced by Charge Delocalization and Vibronically Hot Exciton Dissociation. J. Am. Chem. Soc. 2013, 135, 16364-16367.

(21) Huix-Rotllant, M.; Tamura, H.; Burghard, I. Concurrent Effects of Delocalization and Internal Conversion Tune Charge Separation at Regioregular PolythiopheneFullerene Heterojunctions. J. Phys. Chem. Lett. 2015, 6, 1702-170.

(22) Patel, K.; Bittner, E. R. Mixed Quantum Classical Simulations of Charge-Transfer Dynamics in a Model Light-Harvesting Complex. I. ChargeTransfer Dynamics. J. Phys. Chem. B 2020, 124, 2149-2157.
(23) Patel, K.; Bittner, E. R. Mixed Quantum Classical Simulations of Charge-Transfer Dynamics in a Model Light-Harvesting Complex. II. Transient Vibrational Analysis. J. Phys. Chem. B 2020, 124, 2158-2167.

(24) Giannini, S.; Carof, A.; Ellis, M.; Yang, H.; Ziogos, O. G.; Ghosh, S.; Blumberger, J. Quantum localization and delocalization of charge carriers in organic semiconducting crystals. Nat. Comm. 2019, 10, 3843 .

(25) Carof, A.; Giannini, S.; Blumberger, J. How to calculate charge mobility in molecular materials from surface hopping non-adiabatic molecular dynamics - beyond the hopping/band paradigm. Phys. Chem. Chem. Phys. 2019, 21, 26368.

(26) Zhu, H.; Yang, Y.; Kim, H.-D.; Califano, M.; Song, N.; Wang, Y.; Zhang, W.; Prezhdo, O.; Lian, T. Auger-Assisted Electron Transfer from Photoexcited Semiconductor Quantum Dots. Nano. Lett. 2013, 14, 1263-1269.

(27) Hyeon-Deuk, K.; Prezhdo, O. V. Ab Initio Analysis of Auger-Assisted Electron Transfer. J. Phys. Chem. Lett. 2015, 6, 1263-1269.

(28) Trivedi, D.; Wang, L.; Prezhdo, O. Augermediated electron relaxation is robust to deep hole traps: time-domain ab initio study of CdSe quantum dots. Nano letters 2015, 15, 2086-2091.

(29) Goyal, P.; Hammes-Schiffer, S. Role of Solvent Dynamics in Photoinduced Proton-Coupled Electron Transfer in Phenol-Amine Complex in Solution. J. Phys. Chem. Lett. 2015, 6, 3515-3520.

(30) Goyal, P.; Schwerdtfeger, C. A.; Soudackov, A. V.; Hammes-Schiffer, S. Proton Quantization and Vibrational Relaxation in Nonadiabatic Dynamics of Photoinduced Proton-Coupled Electron Transfer in a Solvated Phenol-Amine Complex. J. Phys. Chem. B 2016, 120, 2407-2417.

(31) Goyal, P.; Hammes-Schiffer, S. Tuning the Ultrafast Dynamics of Photoinduced Proton-Coupled Electron Transfer in Energy Conversion Processes. ACS Energy Lett. 2017, 2, 512-519.

(32) Wang, L.; Long, R.; Prezhdo, O. V. Time-Domain Ab Initio Modeling of Photoinduced Dynamics at Nanoscale Interfaces. Ann. Rev. Phys. Chem. 2015, 66, 549-579.

(33) Tully, J. C. Molecular dynamics with Electronic Transitions. J. Chem. Phys. 1990, 93, 1061-1071.

(34) Pal, S.; Trivedi, D. J.; Akimov, A. V.; Aradi, B.; Frauenheim, T.; Prezhdo, O. V. Nonadiabatic molecular dynamics for thousand atom systems: A 
tight-binding approach toward pyxaid. Journal of chemical theory and computation 2016, 12, 14361448.

(35) Fischer, S. A.; Habenicht, B. F.; Madrid, A. B.; Duncan, W. R.; PrezhdoV, O. V. Regarding the validity of the time-dependent Kohn-Sham approach for electron-nuclear dynamics via trajectory surface hopping. J. Chem. Phys 2011, 134, 024102 .

(36) Liu, X.-Y.; Liu, X.-Y.; Liu, X.-Y.; Li, Z.-W.; Fang, W.-H.; Cui, G. Nonadiabatic Exciton and Charge Separation Dynamics at Interfaces of Zinc Phthalocyanine and Fullerene: Orientation Does Matter. J. Phys. Chem. A 2020, 124, 7388-7398.

(37) Rego, L. G.; Hames, B. C.; Mazon, K. T.; Joswig, J.-O. Intramolecular polarization induces electron-hole charge separation in light-harvesting molecular triads. J. Phys. Chem. C 2014, 118, 126-134.

(38) High, J. S.; Rego, L. G.; Jakubikova, E. Quantum dynamics simulations of excited state energy transfer in a zinc-free-base porphyrin dyad. $J$. Phys. Chem. A 2016, 120, 8075-8084.

(39) Hu, Z.; Tong, Z.; Cheung, M. S.; Dunietz, B. D.; Geva, E.; Sun, X. Photoinduced Charge Transfer Dynamics in the Carotenoid-Porphyrin-C60 Triad via the Linearized Semiclassical Nonequilibrium Fermi's Golden Rule. J. Phys. Chem. B 2020, 124, 9579-9591.

(40) Koch, W.; Holthausen, M. C. A Chemist's Guide to Density Functional Theory; John Wiley \& Sons, 2001.

(41) Akimov, A. V.; Prezhdo, O. V. The PYXAID Program for Non-Adiabatic Molecular Dynamics in Condensed Matter Systems. J. Chem. Theory Comput. 2013, 9, 4959-4972.

(42) Akimov, A.; Prezhdo, O. Theory of Nonadiabatic Electron Dynamics in Nanomaterials. 2015.

(43) Wang, L.; Akimov, A.; Prezhdo, O. V. Recent Progress in Surface Hopping: 2011-2015. J. Phys. Chem. Lett. 2016, 7, 2100-2112.

(44) Doltsinis, N. L.; Marx, D. First principles molecular dynamics involving excited states and nonadiabatic transitions. Journal of Theoretical and Computational Chemistry 2002, 1, 319-349.

(45) Akimov, A. V.; Prezhdo, O. V. Advanced capabilities of the PYXAID program: integration schemes, decoherence effects, multiexcitonic states, and field-matter interaction. J. Chem. Theory Comput. 2014, 10, 789-804.
(46) Craig, C. F.; Duncan, W. R.; Prezhdo, O. V. Trajectory surface hopping in the time-dependent Kohn-Sham approach for electron-nuclear dynamics. Physical review letters 2005, 95, 163001.

(47) Gao, X.; Geng, H.; Peng, Q.; Ren, J.; Yi, Y.; Wang, D.; Shuai, Z. Nonadiabatic Molecular Dynamics Modeling of the Intrachain Charge Transport in Conjugated Diketopyrrolo-Pyrrole Polymers. J. Phys. Chem. C 2014, 118, 6631-6640.

(48) Akimov, A. V. Nonadiabatic Molecular Dynamics with Tight-Binding Fragment Molecular Orbitals. J. Chem. Theory Comput. 2016, 12, 5719-5736.

(49) High, J. S.; Rego, L. G. C.; Jakubikova, E. Quantum Dynamics Simulations of Excited State Energy Transfer in a Zinc-Free-Base Porphyrin Dyad. J. Phys. Chem. A 2016, 120, 8075-8084.

(50) Humeniuk, A.; Mitric, R. DFTBaby: A Software Package for Non-Adiabatic Molecular Dynamics Simulations Based on Long-Range Corrected Tight-Binding TD-DFT(B). 2017,

(51) Parandekar, P. V.; Tully, J. C. Detailed balance in Ehrenfest mixed quantum-classical dynamics. $J$. Chem. Theory Comput. 2006, 2, 229-235.

(52) Subotnik, J. E.; Jain, A.; Landry, B.; Petit, A.; Ouyang, W.; Bellonzi, N. Understanding the Surface Hopping View of Electronic Transitions and Decoherence. Annu. Rev. Phys. Chem. 2016, 67, 387-417.

(53) Smith, B.; Akimov, A. V. A comparative analysis of surface hopping acceptance and decoherence algorithms within the neglect of back-reaction approximation. J. Chem. Phys. 2019, 151, 124107.

(54) Landry, B. R.; Falk, M. J.; Subotnik, J. E. Communication: The correct interpretation of surface hopping trajectories: How to calculate electronic properties. Journal of Chemical Physics 2013, 139, 211101-211101.

(55) Elstner, M.; Porezag, D.; Jungnickel, G.; Elsner, J.; Haugk, M.; Frauenheim, T.; Suhai, S.; Seifert, G. Self-consistent-charge densityfunctional tight-binding method for simulations of complex materials properties. Phys. Rev. B 1998, 58, 7260 .

(56) Porezag, D.; Frauenheim, T.; Köhler, T.; Seifert, G.; Kaschner, R. Construction of tightbinding-like potentials on the basis of densityfunctional theory: Application to carbon. Phys. Rev. B 1995, 51, 12947. 
(57) Gaus, M.; Cui, Q.; Elstner, M. DFTB3: Extension of the Self-Consistent-Charge Density-Functional Tight-Binding Method (SCC-DFTB). J. Chem. Theory Comput. 2011, 7, 931-948.

(58) Scholz, R.; Luschtinetz, R.; Seifert, G.; JägelerHoheisel, T.; Körner, C.; Leo, K.; Rapacioli, M. Quantifying charge transfer energies at donoracceptor interfaces in small-molecule solar cells with constrained DFTB and spectroscopic methods. Journal of physics: Condensed matter 2013, 25, 473201 .

(59) Oviedo, M. B.; Wong, B. M. Real-time quantum dynamics reveals complex, many-body interactions in solvated nanodroplets. J. Chem. Theory Comput. 2016, 12, 1862-1871.

(60) Gaus, M.; Goez, A.; Elstner, M. Parametrization and Benchmark of DFTB3 for Organic Molecules. J. Chem. Theory Comput. 2013, 9, 338-354.

(61) Aradi, B.; Hourahine, B.; Frauenheim, T. DFTB+, a Sparse Matrix-Based Implementation of the DFTB Method. J. Phys. Chem. A 2007, $111,5678-5684$.

(62) Rappe, A. K.; Casewit, C. J.; Colwell, K. S.; Goddard, W. A.; Skiff, W. M. UFF, a Full Periodic Table Force Field for Molecular Mechanics and Molecular Dynamics Simulations. J. Am. Chem. Soc. 1992, 114, 10024-10035.

(63) Torres, A.; Oliboni, R. S.; Rego, L. G. Vibronic and Coherent Effects on Interfacial Electron Transfer Dynamics. J. Phys. Chem. Lett. 2015, 6, 4927-4935.

(64) Hammes-Schiffer, S.; Tully, J. C. Proton Transfer in Solution: Molecular Dynamics with Quantum Transitions. J. Chem. Phys. 1994, 12, 4657-4667.

(65) Space, B.; Coker, D. Nonadiabatic Dynamics of Excited Excess Electrons in Simple Fluids. $J$. Chem. Phys. 1991, 94, 1976-1984.

(66) Yu, N.; Margulis, C.; Coker, D. Influence of Solvation Environment on Excited State Avoided Crossings and Photodissociation Dynamics. J. Phys. Chem. B 2001, 105, 6728-6737.

(67) Hu, C.; Sugino, O.; Hirai, H.; Tateyama, Y. Nonadiabatic couplings from the Kohn-Sham derivative matrix: Formulation by time-dependent densityfunctional theory and evaluation in the pseudopotential framework. Phys. Rev. A 2010, 82, 062508.

(68) Mandal, A.; Yamijala, S. S.; Huo, P. QuasiDiabatic Representation for Nonadiabatic Dynamics Propagation. J. Chem. Theory Comput. 2018, 14, 1828-1840.
(69) Long, R.; English, N. J.; Prezhdo, O. V. Photoinduced Charge Separation across the GrapheneTiO2 Interface Is Faster than Energy Losses: A Time-Domain ab Initio Analysis. J. Am. Chem. Soc. 2012, 134, 14238-14248.

(70) Liddell, P. A.; Kuciauskas, D.; Sumida, J. P.; Nash, B.; Nguyen, D.; Moore, A. L.; Moore, T. A.; Gust, D. Photoinduced charge separation and charge recombination to a triplet state in a carotene- porphyrin- fullerene triad. Journal of the American Chemical Society 1997, 119, 14001405.

(71) Gust, D.; Moore, T. A.; Moore, A. L. Mimicking photosynthetic solar energy transduction. Accounts of Chemical Research 2001, 34, 40-48.

(72) da Silva Oliboni, R.; Bortolini, G.; Torres, A.; Rego, L. G. C. A Nonadiabatic Excited State Molecular Mechanics/Extended Hückel Ehrenfest Method. J. Phys. Chem. C. 2016, 120, 2768827698 .

(73) Nijjar, P.; Jankowska, J.; Prezhdo, O. V. Ehrenfest and classical path dynamics with decoherence and detailed balance. J. Chem. Phys. 2019, 150, 204124 . 